\title{
ON THE GROUP OF CONFORMAL TRANSFORMATIONS OF A COMPACT RIEMANNIAN MANIFOLD. III
}

\author{
CHUAN-CHIH HSIUNG
}

\section{Introduction}

Let $g_{i j}, R_{h i j k}, R_{i j}=R_{i j k}^{k}$ be respectively the metric, Riemann and Ricci tensors of a Riemannian manifold $M^{n}$ of dimension $n$, and denote

$$
P=R^{h i j k} R_{h i j k}, \quad Q=R^{i j} R_{i j} .
$$

Throughout this paper all Latin indices take the values $1, \cdots, n$ unless stated otherwise, and repeated indices imply summation. In a recent paper [2] the author proved

Theorem 1. Suppose that a compact Riemannian manifold $M^{n}(n>2)$ with constant scalar curvature $R=g^{i j} R_{i j}$ admits an infinitesimal nonhomothetic conformal transformation $v$, and let $L_{v}$ be the operator of the infinitesimal transformation $v$. If

$$
a^{2} L_{v} P+b(2 a+n b) L_{n} Q=\text { const., }
$$

where $a$ and $b$ are constants such that

$$
c \equiv 4 a^{2}+2(n-2) a b+n(n-2) b^{2}>0,
$$

then $M^{n}$ is isometric to a sphere.

In particular, when $a=0$ or $b=0$, Theorem 1 is reduced to a result of Yano [4], which is a generalization of some results of Lichnerowicz [3] and the author [1]. Yano pointed out that condition (1.3) is equivalent to that $a$ and $b$ are not both zero.

Very recently, Yano and Sawaki [5] obtained the following theorem similar to Theorem 1:

Theorem 2. Suppose that a compact Riemannian manifold $M^{n}(n>2)$ with constant $R$ admits an infinitesimal nonhomothetic conformal transformation v. If

$$
L_{v} L_{v}\left[(n-2) a^{2} P+4 b(2 a+b) Q\right] \leq 0,
$$

Communicated November 28,1967 . Supported partially by the National Science Foundation grant GP-7513. 
where $a$ and $b$ are constants such that $a+b \neq 0$, then $M^{n}$ is isometric to $a$ sphere.

The purpose of this paper is to generalize both Theorems 1 and 2 to

Theorem 3. Suppose that a compact Riemannian manifold $M^{n}(n>2)$ with constant $R$ admits an infinitesimal nonhomothetic conformal transformation v. If

$$
L_{v} L_{v}\left(a^{2} P+\frac{c-4 a^{2}}{n-2} Q\right) \leq 0
$$

where

$$
\begin{aligned}
c \equiv 4 a^{2}+(n-2)[ & 2 a \sum_{i=1}^{4} b_{i}+\left(b_{1}-b_{2}+b_{3}-b_{4}+b_{5}-b_{6}\right)^{2} \\
& \left.-2 b_{1} b_{3}-2 b_{2} b_{4}+2 b_{5} b_{6}+(n-1) \sum_{i=1}^{6} b_{1}^{2}\right]>0,
\end{aligned}
$$

$a$ and $b^{\prime} s$ being constants, then $M^{n}$ is isometric to a sphere.

It is obvious that Theorem 3 is reduced to a generalization of Theorem 1 when $b_{2}=\cdots=b_{6}=0$, and to Theorem 2 when

$$
b_{1}=\cdots=b_{4}=b /(n-2), \quad b_{5}=b_{6}=0 .
$$

We need the following theorem of Yano [5] to prove Theorem 3:

Theorem 4. Suppose that a compact orientable Riemannian manifold $M^{n}$ $(n>2)$ with constant $R$ admits an infinitesimal nonhomothetic conformal transformation $v$ so that

$$
L_{v} g_{i j}=2 \phi g_{i j}, \quad \phi=\text { const },
$$

and let $\nabla$ denote the operator of covariant derivation of $M^{n}$ with respect to $g_{i j}$. If

$$
\int_{M^{n}} T_{i j} \phi^{i} \phi^{j} d A \geq 0
$$

where

$$
T_{i j}=R_{i j}-\frac{R}{n} g_{i j},
$$

$\phi^{i}=\nabla^{i} \phi=g^{i j} \nabla_{j} \phi$, and $d A$ is the element of area of $M^{n}$ at a point, then $M^{n}$ is isometric to a sphere.

\section{Lemmas}

Throughout this section $M^{n}$ will always denote a compact orientable Riemannian manifold of dimension $n>2$. Let $\Delta$ be the Laplace-Beltrami operator on $M^{n}$. Then, for any scalar field $f$ on $M^{n}$, 


$$
\Delta f=-\nabla^{i} \nabla_{j} f .
$$

Thus we have

$$
\int_{M^{n}} \Delta f d A=0
$$

from the well-known Green's formula:

$$
\int_{M^{n}} \nabla^{i} \xi_{i} d A=0,
$$

where $\xi_{i}$ is any vector field on $M^{n}$.

Lemma 1. If a nonconstant scalar field $\phi$ on a manifold $M^{n}$ satisfies $\Delta \phi=$ $k \phi$, where $k$ is constant, then $k$ is positive.

Proof. From equations (2.1), (2.2) we obtain

$$
\begin{aligned}
0=\int_{M^{n}} \Delta\left(\phi^{2}\right) d A & =2 \int_{M^{n}}\left(\phi \Delta \phi-\phi^{i} \phi_{i}\right) d A \\
& =2 \int_{M^{n}}\left(k \phi^{2}-\phi^{i} \phi_{i}\right) d A,
\end{aligned}
$$

which gives Lemma 1 immediately.

Lemma 2. Let $v$ be an infinitesimal conformal transformation on $M^{n}$ so that

$$
L_{v} g_{i j}=2 \phi g_{i j}
$$

Then

$$
\begin{gathered}
L_{v} R_{h i j k}=2 \phi R_{h i j k}-g_{h k} \nabla_{j} \phi_{i}+g_{h j} \nabla_{k} \phi_{i} \\
-g_{i j} \nabla_{k} \phi_{h}+g_{i k} \nabla_{j} \phi_{h} \\
L_{v} R_{i j}=g_{i j} \Delta \phi-(n-2) \nabla_{j} \phi_{i} \\
L_{v} R=2(n-1) \Delta \phi-2 R \phi .
\end{gathered}
$$

Lemma 2 can be proved by a straightforward computation.

Lemma 3. If $M^{n}$ has constant $R$ and admits an infinitesimal nonhomothetic conformal transformation $v$ so that (1.7) holds, then

$$
\begin{gathered}
\Delta \phi=R \phi /(n-1), \\
R>0 .
\end{gathered}
$$


Equation (2.9) follows from equation (2.8) due to the constancy of $R$, and equation (2.10) from Lemma 1.

Lemma 4 (Yano and Sawaki [5]). If $M^{n}$ admits an infinitesimal conformal transformation $v$ so that equation (2.5) holds, then, for any scalar field $f$ on $M^{n}$,

$$
\int_{M^{n}} \phi f d A=-\frac{1}{n} \int_{M^{n}} L_{v} f d A .
$$

Proof. Substituting $f v_{i}$ for $\xi_{i}$ in the Green's formula (2.3) we obtain

$$
\int_{M^{n}} f \nabla^{i} v_{i} d A=-\int_{M^{n}} v_{i} \nabla^{i} f d A=-\int_{M^{n}} L_{v} f d A .
$$

On the other hand, since

$$
L_{v} g_{i j}=\nabla_{i} v_{j}+\nabla_{j} v_{i},
$$

from equation (2.5) we have $\nabla^{i} v_{i}=n \phi$, which and equation (2.12) yield the required equation (2.11) immediately.

\section{Proof of Theorem 3}

On the manifold $M^{n}$ consider the covariant tensor field of order 4:

$$
\begin{aligned}
W_{h i j k}= & a T_{h i j k}+b_{1} g_{h k} T_{i j}-b_{2} g_{h j} T_{i k}+b_{3} g_{i j} T_{h k} \\
& -b_{4} g_{i k} T_{h j}+b_{5} g_{h i} T_{j k}-b_{6} g_{j k} T_{h i},
\end{aligned}
$$

where

$$
T_{h i j k}=R_{h i j k}-\frac{R}{n(n-1)}\left(g_{i j} g_{h k}-g_{i k} g_{h j}\right)
$$

and $a$ and $b$ 's are constants satisfying (1.6). Then

$$
W^{h i j k} W_{h i j k}=a^{2} P+\frac{c-4 a^{2}}{n-2} Q-\frac{1}{n}\left(\frac{2 a^{2}}{n-1}+\frac{c-4 a^{2}}{n-2}\right) R^{2},
$$

where $c$ is defined by (1.6). From equation (3.3) it follows that

$$
L_{v}\left(W^{h i j k} W_{h i j k}\right)=L_{v}\left(\left(a^{2} P+\frac{c-4 a^{2}}{n-2} Q\right) .\right.
$$

By assuming the infinitesimal nonhomothetic conformal transformation $v$ to 
be defined by (1.7), from equations (3.1), (3.2), (1.9), (2.5), (2.6), (2.7), (2.9) we can easily obtain

$$
\begin{aligned}
L_{v} W_{h i j k}= & 2 a \phi R_{h i j k}-\left[a+(n-2) b_{1}\right] g_{h k} \nabla_{j} \phi_{i} \\
& +\left[a+(n-2) b_{2}\right] g_{h j} \nabla_{k} \phi_{i}-\left[a+(n-2) b_{3}\right] g_{i j} \nabla_{k} \phi_{h} \\
& +\left[a+(n-2) b_{4}\right] g_{i k} \nabla_{j} \phi_{h}-(n-2) b_{5} g_{h i} \nabla_{k} \phi_{j}+(n-2) b_{6} g_{j k} \nabla_{i} \phi_{h} \\
& -\frac{\phi R}{n(n-1)} g_{i j} g_{h k}\left[4 a+(3 n-4)\left(b_{1}+b_{3}\right)\right] \\
& +\frac{\phi R}{n(n-1)} g_{i k} g_{h j}\left[4 a+(3 n-4)\left(b_{2}+b_{4}\right)\right] \\
& -\frac{3 n-4}{n(n-1)} b_{5} \phi R g_{h i} g_{j k}+\frac{3 n-4}{n(n-1)} b_{6} \phi R g_{j k} g_{h i}+2 b_{1} \phi g_{h k} R_{i j} \\
& -2 b_{2} \phi g_{h j} R_{i k}+2 b_{3} \phi g_{i j} R_{h k}-2 b_{4} \phi g_{i k} R_{h j}+2 b_{5} \phi g_{h i} R_{j k} \\
& -2 b_{6} \phi g_{j k} R_{k i} .
\end{aligned}
$$

Multiplying both sides of equation (3.5) by $W^{\text {hijk }}$ and making use of equations (3.1), (3.2), (1.9), (3.3), (1.6) and $R_{i j k}^{i}=0$, an elementary but lengthy calculation yields

$$
W^{h i j k} L_{n} W_{h i j k}=2 \phi W^{h i j k} W_{h i j k}-c T^{i j} \nabla_{j} \phi_{i} .
$$

By substituting equation (3.6) in the well-known formula

$$
L_{v}\left(W^{h i j k} W_{h i j k}\right)+2 W^{h i j k} L_{v} W_{h i j k}-8 \phi W^{h i j k} W_{h i j k},
$$

we thus have

$$
\phi L_{v}\left({ }^{h i j k} W_{h i j k}\right)=-4 \phi^{2} W^{h i j k} W_{h i j k}-2 c \phi T^{i j} \nabla_{\jmath} \phi_{i} .
$$

Since the manifold $M^{n}$ is of constant $R$, it is known that fo $n<2$

$$
\nabla^{j} R_{i j}=0
$$

and therefore

$$
\nabla^{j} T_{i j}=0
$$

Thus

$$
\nabla^{j}\left(T_{i j} \phi \phi^{i}\right)=T_{i j} \phi^{i} \phi^{j}+\phi T_{i j} \nabla^{j} \phi^{i}
$$

Without loss of generality we may assume our manifold $M^{n}$ to be orientable, since otherwise we need only to take an orientable twofold covering space of 
$M^{n}$. Substituting equation (3.8) in equation (3.11), integrating the resulting equation over the manifold $M^{n}$ and using equation (2.3) we obtain

$$
\begin{aligned}
2 c \int_{M^{n}} T_{i j} \phi^{i} \phi^{j} d A= & \int_{M^{n}} \phi L_{v}\left(W^{h i j k} W_{h i j k}\right) d A \\
& +4 \int_{M^{n}} \phi^{2} W^{h i j k} W_{h i j k} d A .
\end{aligned}
$$

On the right side of equation (3.12), the second integral is nonnegative since its integrand is so, and the first integral is equal to, by Lemma 4 and equations (3.3), (1.5),

$$
-\frac{1}{n} \int_{M^{n}} L_{v} L_{v}\left(W^{h i j k} W_{h i j k}\right) d A=-\frac{1}{n} \int_{M^{n}} L_{v} L_{v}\left(a^{2} P+\frac{c-4 a^{2}}{n-2} Q\right) d A \geq 0 .
$$

Hence the integral on the left side of equation (3.12) is nonnegative, and Theorem 3 follows from Theorem 4 immediately.

\section{References}

[1] C.C.Hsiung, On the group of conformal transformations of a compact Riemannian manifold, Proc. Nat. Acad. Sci. U.S.A. 54 (1965) 1509-1513.

[2] - On the group of conformal transformations of a compact Riemannian manifold. II, Duke Math. J. 34 (1967) 337-341.

[3] A. Lichnerowicz, Sur les transformations conformes d'une variété riemannienne compacte, C. R. Acad. Sci. Paris 259 (1964) 697-700.

[4] K. Yano, On Riemannian manifolds with constant scalar curvature admitting a conformal transformation group, Proc. Nat. Acad. Sci. U.S.A. 55 (1966) 472-476.

[5] K. Yano \& S. Sawaki, Riemannian manifolds admitting a conformal transformation group, J. Differential Geometry 2 (1968) 185-190.

LEHIGH UNIVERSITY 